\title{
Research on caregiver stress: time for integration and application
}

Over the past two decades, caregiver stress has been the subject of scientific inquiry by investigators around the world representing a variety of disciplines. The knowledge base is extensive and growing, particularly as it relates to the phenomenology and correlates of caregiver stress. The six papers published here represent a snapshot of prevailing lines of inquiry and enhance our knowledge regarding: predictors of psychological distress for dementia family caregivers in Japan (Shikimoto et al., 2017) and for Indonesian and Burmese foreign domestic workers caring for frail older adults in Singapore (Ha et al., 2018); identification of a threshold number of behavioral symptoms in those with dementia associated with caregiver distress (Arthur et al., 2017); the influence of specific illness factors on caregiver stress, including comorbid diabetes in patients with Alzheimer's disease ( $\mathrm{Li}$ et al., 2018) and a comparison of the effects of behavioral disturbances on caregiver burden across three types of dementia (Liu et al., 2017); and the application of data on caregiver stress to inform the development of a multicomponent framework for preventing depression in caregivers of those with dementia (Ying et al., 2017).

Through these studies of caregivers from different countries and cultures, these teams of investigators have added confirmatory support to the following notions about caregiver stress. First, caregiver stress is a costly, often life-altering phenomenon experienced by both informal and professional caregivers worldwide. Second, there is no one universal caregiving experience as each is shaped by innumerable factors specific to the caregiver, care receiver, illness characteristics, and sociocultural context. And, third, the knowledge base on caregiver stress has grown sufficiently to inform much needed development of meaningful interventions.

While there is now strong consensus on the causes and correlates of stress, the increasing number of published review papers on caregiver stress and burden in recent years suggests that there is a burgeoning scientific quest, of sorts, striving to interpret and organize the voluminous data from previous reports. Review papers have focused on identifying synthesizing constructs, promoting use of consistent and psychometrically sound measures, and extracting clinical significance of findings all in the service of intervention development to prevent or alleviate caregiver stress. With this in mind, and a focus on this collection of papers, I offer the following reflections to inform next steps.

\section{Value of synthesizing constructs and specificity of measures}

The stress process model proposed by Pearlin et al. (1990) provides a framework that remains useful in conceptualizing and interpreting caregiver stress. In this model, caregiver stress is viewed as a consequence of key interrelated factors, including the caregiver's socioeconomic characteristics, resources such as coping mechanisms and social support, and the primary and secondary stressors to which they are exposed. Primary stressors are those that arise specifically from caregiving. Secondary stressors are those that emanate from roles and activities external to caregiving, as well as intrapsychic strains such as self-esteem, mastery, and loss of self that often impair a caregiver's sense of well-being. These stressors contribute to a caregiver's sense of burden. Objective burden measures the tasks and hours involved in caregiving. Subjective burden captures how the caregiver perceives the experience of providing care as well as the emotional impact of caregiving in its various forms.

Application of the stress process model to the papers featured herein adds unifying threads regarding caregiver stress and raises questions for further study. For example, Shikimoto et al. (2017) reported that psychological distress of caregivers in Japan is high (subjective burden) and that the degree of distress is associated with the amount of informal care time provided (objective burden), the number of caregivers (resources), and the number and nature of the behavioral and psychological symptoms of dementia. While the number of symptoms best characterizes objective burden, the "nature" of symptoms is less clear in that it could be further described in terms of the tasks or time 
associated with symptom management (objective), or the impact of the symptoms on caregiver's well-being, or on the interpersonal relationship between the caregiver and care receiver (subjective burden).

Arthur et al. (2017) studied dyads of care receivers with dementia and their caregivers in an effort to identify if there was a threshold of behavioral and psychological symptoms of dementia that was predictive of caregiver depression and burden. In this study, the notion of "threshold" itself speaks to subjective burden whereas the tasks associated with management of the symptoms would contribute to objective burden and the caregiver's experience of the symptoms would likely contribute to subjective burden, particularly if they triggered emotional responses or other types of distress.

$\mathrm{Li}$ et al. (2018) set out to examine whether a comorbid condition such as diabetes in a care receiver added to caregiver stress as measured by the Zarit Burden Interview (ZBI). In addition to total ZBI score, investigators also looked at ZBI factors, including effect on the social and personal life of caregivers (objective burden), the psychological burden (subjective burden), and feelings of guilt (subjective burden). Similarly, Liu et al. (2017) studied Chinese caregivers of care receivers with frontotemporal lobar dementia, Lewy Body dementia, or Alzheimer's disease to compare measures of burden, sleep quality, depression, and anxiety. They measured burden using the total ZBI score and specific measures for sleep, depression, and anxiety. While the composite ZBI score does not allow us to isolate more specific types of burden, it does provide comparison of differences in magnitude of burden across caregiver samples. In the stress process model, measures of sleep, depression, and anxiety can represent manifestations of subjective burden related to caregiving but can also be interpreted as manifestations of secondary stressors or coping styles.

\section{Importance of relational and contextual factors}

The exercise of applying the findings from these papers to the stress process model punctuated for me the void of information we have available to us on relational and contextual factors regarding caregivers in study populations. Family characteristics and other sociocultural factors largely influence the family's experience of life with dementia as well as health outcomes for the person with dementia and other family members (Podgorski, 2017). Illnesses such as dementia disrupt the normal trajectory of the family life cycle with ripple effects across all generations of the family in domains, including family function, interpersonal relationships, caregiver health, and financial stability.

Every family has a past, a present, and some vision of the future. Families move through life cycle stages and are confronted with the tasks and responsibilities associated with each stage. As a family transitions through time, its members carry with them "vertical stressors" and encounter "horizontal stressors." Vertical stressors, such as family secrets, attitudes, health beliefs, gender roles, and rules regarding expression of emotions, for example, are emotional norms and rules transmitted across generations. Horizontal stressors are predictable, developmental events such as births, marriages, retirement, and deaths as well as unpredictable events such as divorce or illness. Being a caregiver is rarely the only stressor in a person's life. These other stressors constitute those that comprise the "secondary stressors" and add to "subjective burden" in the stress process model. A recent publication by Chen et al. (2017) illustrates the importance of understanding relational dynamics in caregiving. These investigators found that daughters' beliefs that their parent can control their dementiarelated symptoms were associated with more relationship conflict or high expressed emotion. The authors concluded that daughters' beliefs about parental behavior may contribute to caregiver stress and exacerbate negative behaviors exhibited by individuals with dementia.

Research on caregivers rarely considers such contextual factors. While caregiver-care receiver dyads are often studied, the information reported on each is usually limited to sociodemographic variables. The nature and quality of the relationship between the two is seldom captured yet strongly influences the caregiving and care receiving experiences. One's motivation for caregiving (e.g. attachment, obligation, or exchange), for example, has long been associated with health outcomes yet is rarely included. And it is well established that family conflict diminishes the health and quality of life for persons with dementia and their family caregivers (Podgorski, 2017). Key relational dynamics worth consideration include attachment style, balance of power, quality of relationships, role delineation, expressed emotion, communication, and problem solving abilities. In addition, previous family experiences of abandonment, trauma, or infidelity, for example, strongly influence the motivation for and commitment to caregiving as well as the boundaries caregivers will establish when assuming a caregiving role. 


\section{From predictors and correlates to interventions}

The body of knowledge on caregiver stress is considerable and, in response to the growing recognition of the tremendous societal costs of caregiver burden, there is urgency to hasten the transition from research on correlates to interventions to alleviate distress. The explicit adoption of and adherence to a shared conceptual framework would serve to enhance collaboration among investigators worldwide and to expand the capacity to compare, interpret, and integrate findings from across disciplines. In addition, an increased understanding of the caregiver's relational and contextual factors is critical to enhance our comprehension of the interpersonal and psychological complexities that contribute to subjective burden.

\section{Carol Podgorski}

University of Rochester Medical Center, 300

Crittenden Boulevard - Box Psychiatry, Rochester, New York 14642 USA.

Email: carol_podgorski@urmc.rochester.edu

\section{References}

Arthur, P., Gitlin, L., Kairalla, J. and Mann, W. (2017). Relationship between the number of behavioral symptoms in dementia and caregiver distress: what is the tipping point?. International Psychogeriatrics, 30, 1099-1107.
Chen, C. K., Clayton, K. and Chodosh, J. (2017). The relationship between "what we believe" and "how we care" among daughters caring for a parent with dementia. American fournal of Alzheimers Disease and Other Dementias, 32, 90-95.

Ha, N., Chong, M., Choo, R., Tam, W. and Yap, P. (2018). Caregiving burden in foreign domestic workers caring for frail older adults in Singapore. International Psychogeriatrics, 30, 1139-1147.

Li, J. et al. (2018). Impact of diabetes on caregiver stress in patients with Alzheimer's disease: data from the ICTUS study. International Psychogeriatrics, 30, 11091117.

Liu, S. et al. (2017). Caregiver burden, sleep quality, depression, and anxiety in dementia caregivers: a comparison of frontotemporal lobar degeneration, dementia with Lewy bodies, and Alzheimer's disease. International Psychogeriatrics, 30, 11311138.

Pearlin, L. I., Mullan, J. T., Semple, S. J. and Skaff, M. M. (1990). Caregiving and the stress process: an overview of concepts and their measures. Gerontologist, 30, 583-594.

Podgorski, C. (2017). Neurocognitive disorders: systemic functionality. In J. A. Russo, J. K. Coker, and J. H. King (eds.), DSM-5 and Family Systems (pp. 443-472). New York: Springer Publishing Company.

Shikimoto, R. et al. (2017). Predictive factors associated with psychological distress of caregivers of people with dementia in Japan: a cross-sectional study. International Psychogeriatrics, 30, 1089-1098.

Ying, J., Yap, P., Gandhi, M. and Liew, T. (2017). Iterating a framework for the prevention of caregiver depression in dementia: a multi-method approach. International Psychogeriatrics, 30, 1119-1130. 